\title{
Simulation of Ionizing/Displacement Synergistic Effects on NPN Bipolar Transistors Irradiated by Mixed Neutrons and Gamma Rays
}

\author{
Yuhao Shan, Yanfei Liu $(\mathbb{D}$, Hao Zheng, and Zheng Peng \\ Xi'an High-Tech Research Institute, Xi'an 710025, China \\ Correspondence should be addressed to Yanfei Liu; bbmcu@126.com
}

Received 25 August 2021; Accepted 7 January 2022; Published 19 January 2022

Academic Editor: Jariah Mohamad Juoi

Copyright @ 2022 Yuhao Shan et al. This is an open access article distributed under the Creative Commons Attribution License, which permits unrestricted use, distribution, and reproduction in any medium, provided the original work is properly cited.

\begin{abstract}
Transistors working in complex radiation environments such as space are simultaneously irradiated by neutrons and gamma rays. But the mechanism of the synergistic radiation effect between the two rays is still unclear. Based on TCAD, the synergistic radiation effects of ionizing/displacement damage caused by mixed neutrons and gamma rays are simulated. The results demonstrate that the synergistic effects are more serious than the simple sum of the two radiation effects due to their mutual enhancement. The change of the carrier recombination rate in the device at different positions shows that the displacement effects increase the peak value of surface recombination rate; meanwhile, the ionizing dose effects enhance the recombination process in bulk silicon. The mechanism of this phenomenon is that positive charges from the oxide layer and interface enhance the recombination of carriers in bulk, and the reduced carrier lifetime caused by defects from bulk makes carriers more likely to be trapped by the interface traps. In addition, the simulation result which shows the influence of temperature on the synergistic effects indicates that the synergistic effects are more sensitive to the lower temperature.
\end{abstract}

\section{Introduction}

Although complementary metal oxide semiconductor (CMOS) devices have an absolute advantage in all kinds of microelectronic products because of low-power dissipation, bipolar junction transistors (BJT) still have a wide range of applications due to their inherent ultra-high-speed characteristics and superior capabilities of analog signal processing. When a transistor works in a radiation environment such as space environment, all kinds of radiation effects can damage the transistor and reduce its life span or even cause it to fail such as total ionization dose (TID) effects, displacement damage (DD) effects, and single event effects (SEE). Instead of studying each single radiation effect, recent research has begun to focus on the combined effect of multiple radiation effects, and it has been discovered that bipolar transistors exhibit certain synergistic effects when the ionizing damage and the displacement damage both exist in BJT.
$\mathrm{Li}$ et al. irradiated bipolar transistors with different fluences of $6 \mathrm{MeV}$ carbon ions and $70 \mathrm{keV}$ electrons, which can cause displacement damage and ionization damage, respectively. Then, they tested the deep-level transient spectrum of defects and found that the two types of damages are not working individually $[1,2]$. Based on simulations and experiments, Wang et al. discovered the gain degradation caused by mixed neutrons and gamma rays on lateral PNP transistors is greater than the simple sum of the DD and TID [3-5]. They all illustrated that TID effects could enhance DD effects. However, our research found that this is not only the case, and the DD effects will also have an impact on TID effects.

In this paper, a three-dimensional model of vertical NPN transistor is established with TCAD software to explore the synergistic effects of TID and DD on the NPN BJT caused by gamma rays and neutrons. The mechanism is explored by analyzing the recombination rate of carriers. In addition, the 
sensitivity of the device's working temperature on the synergistic effects is studied as well.

\section{Device Model and Simulation Method}

2.1. Device Modeling. Vertical NPN transistors are widely used in actual ultra-high-speed bipolar integrated circuits. The three-dimensional NPN transistor model constructed with TCAD software is shown in Figure 1 (the unit of length is $\mu \mathrm{m}$ ). The substrate is made of low-doped p-type silicon. A layer of buried subcollector area with higher doping is set at the bottom of the lower-doped collector area to increase the $\mathrm{B}-\mathrm{C}$ junction breakdown voltage, reduce the barrier capacitance, and cut down the $\mathrm{B}-\mathrm{C}$ junction series connection resistance. The top of the transistor uses $\mathrm{SiO}_{2}$ as the insulating protective layer. The material of the emitter and collector in contact with the electrode is polysilicon. Specific doping information of each area is marked in Figure 1 ("-" means p-type doping).

2.2. Simulation Method. Based on previous research results, TCAD software has set the drift, diffusion, generation, and recombination of carriers in the device as different preset models so that researchers can call them directly. For NPN transistors, mobility models with doping dependence and high field saturation and recombination model of SRH (Shockley-Read-Hall) and Auger are required. In simulation, the results of the device performance can be obtained by solving the Poisson equation about the electric potential and the carrier continuity equations. The Gummel curve is the variation of the base current $I_{\mathrm{B}}$ and the collector current $I_{\mathrm{C}}$ versus $V_{\mathrm{BE}}$, reflecting the characteristic performance of a transistor. At the initial stage of simulation, the base voltage and collector voltage are set to $0 \mathrm{~V}$, respectively, and the $V_{\mathrm{BE}}$ scans from $-1.2 \mathrm{~V}$ to $0 \mathrm{~V}$. The gain $\beta$ of transistors can be extracted from the Gummel curve, the relative degradation of which is proportional to the excess base current, expressing as $\Delta(1 / \beta)$.

The region of ionization damage caused by gamma rays contains most of the oxide layer and $\mathrm{Si} / \mathrm{SiO}_{2}$ interface. Therefore, while using the built-in radiation model of TCAD, corresponding traps are added to the oxide layer and interface to simulate the oxide positive charges $N_{o t}$ and interface traps $N_{i t}$ induced by ionizing radiation, which can promote surface recombination of carriers.

Displacement damages caused by neutrons are the lattice defects such as vacancy and cluster, which are formed after neutrons knocking atoms away from the apex of the lattice and result in a decrease of carrier lifetime in the bulk Si. The relationship between the neutron fluence $\Phi$ and the carrier lifetime $\tau$ is as follows [6]:

$$
\frac{1}{\tau_{r}}=\frac{1}{\tau_{r 0}}+\frac{\Phi}{K_{r}} .
$$

In formula (1), $\tau_{r}$ and $\tau_{r 0}$, respectively, represent the carrier lifetime after and before neutron irradiation, $K_{r}$ is the radiation damage coefficient, for neutrons with energy higher than $10 \mathrm{keV}, K_{r}=7 \times 10^{6} \mathrm{~s} / \mathrm{cm}^{3}$ [7]. In TCAD, the

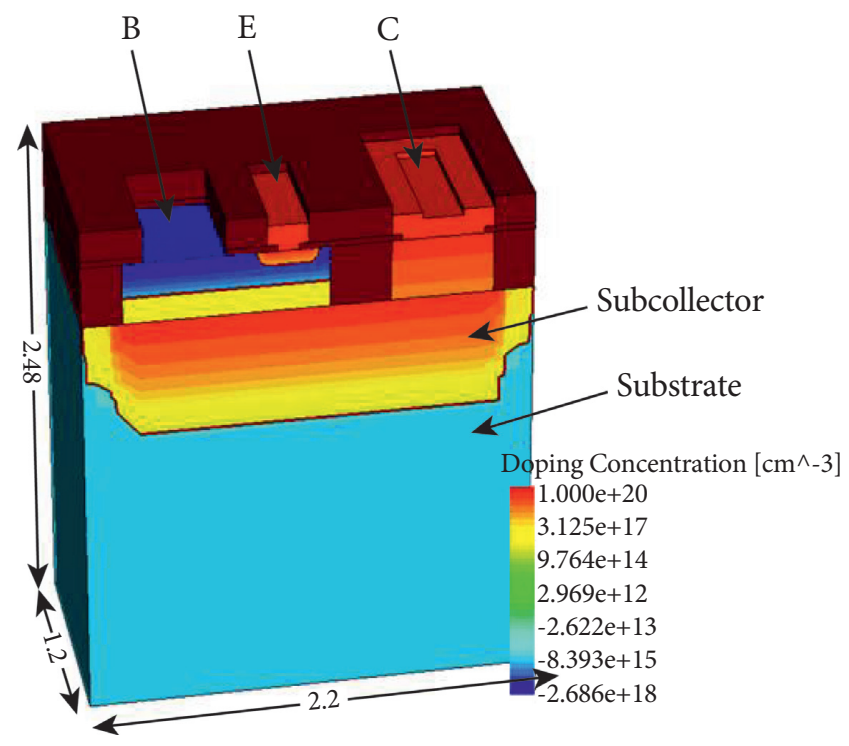

Figure 1: 3D model of the NPN transistor.

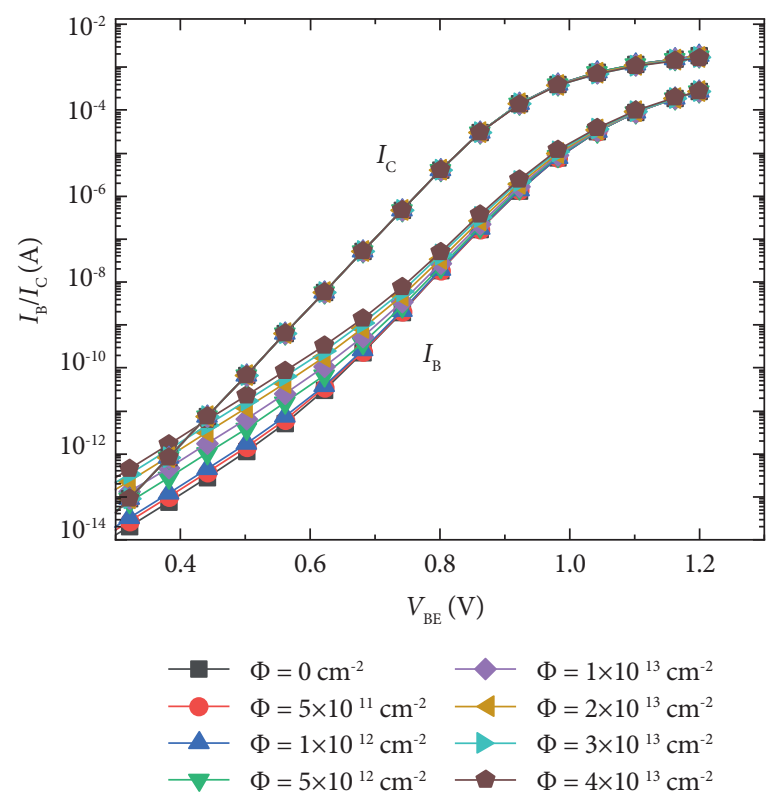

FIgURE 2: Gummel characteristics of NPN BJT after neutron radiation.

carrier lifetime after irradiation calculated by formula (1) is utilized to simulate the influence of displacement damage on transistor performance.

\section{Results and Discussion}

3.1. TID and DD Simulation Results. The Gummel curves of the model transistor changing with different neutron fluences and doses of gamma rays irradiation are shown in Figures 2 and 3. Either the total dose or irradiation fluence increases, the base current $I_{\mathrm{B}}$ increases significantly, and $\Delta I_{\mathrm{B}}$ gradually decreases with the increase of $V_{\mathrm{BE}}$, nearly 0 when $V_{\mathrm{BE}}=1.2 \mathrm{~V} ; I_{\mathrm{C}}$ remains unchanged. It seems that the DD effects caused by neutron irradiation are more serious than 




FIGURE 3: Gummel characteristics of NPN BJT after gamma rays radiation.

the TID effects produced by gamma rays. Both are in line with the results of the actual radiation experiment which contains the $\mathrm{C}$ ion $\mathrm{DD}$ experiment of $\mathrm{Li}$ et al. and the ${ }^{60} \mathrm{Co}$ gamma ray TID experiment of Olarewaju et al. $[1,8]$, indicating that the simulation method is reliable and effective.

3.2. Synergistic Effect Simulation Results. The synergistic effects of neutron and gamma rays simultaneously irradiating the NPN transistor are simulated, and the results are shown in Figure 4 . Extracting $\Delta(1 / \beta)$ of $V_{\mathrm{BE}}=0.75 \mathrm{~V}$ from the Gummel curve, it is observed that $\Delta(1 / \beta)$ caused by neutron irradiation has a linear relationship with the neutron fluence, which is consistent with the Messenger-Spratt equation [6]. Fixing the total dose of gamma rays in $100 \mathrm{krad}$ $\left(\mathrm{SiO}_{2}\right)$ and increasing the neutron fluence, gain degradation $\Delta(1 / \beta)$ of the synergistic effects is displayed. It is found that when the neutron fluence is low, there is no apparent difference between the result of the coordinated irradiation and the simple sum of the TID and DD; with the increase of the fluence, the gain degradation $\Delta(1 / \beta)$ caused by the ionizing/ displacement synergistic effects is higher than the simple artificial sum of the two effects, which shows that the two kinds of radiation effects have certain synergistic effects. And, the gain attenuation of the transistor irradiated by neutrons and gamma rays at the same time is heavier.

Figure 5 shows $\Delta(1 / \beta)$ of the transistor versus the coirradiation of different neutron fluences and different doses of gamma rays. The gain degradation of the NPN transistor is more affected by the displacement damage caused by neutron irradiation. To a certain extent, the figure contains the corresponding results of transistors irradiated by high-energy protons, heavy ions, and other irradiated particles that can cause displacement damage and ionization damage simultaneously [9].

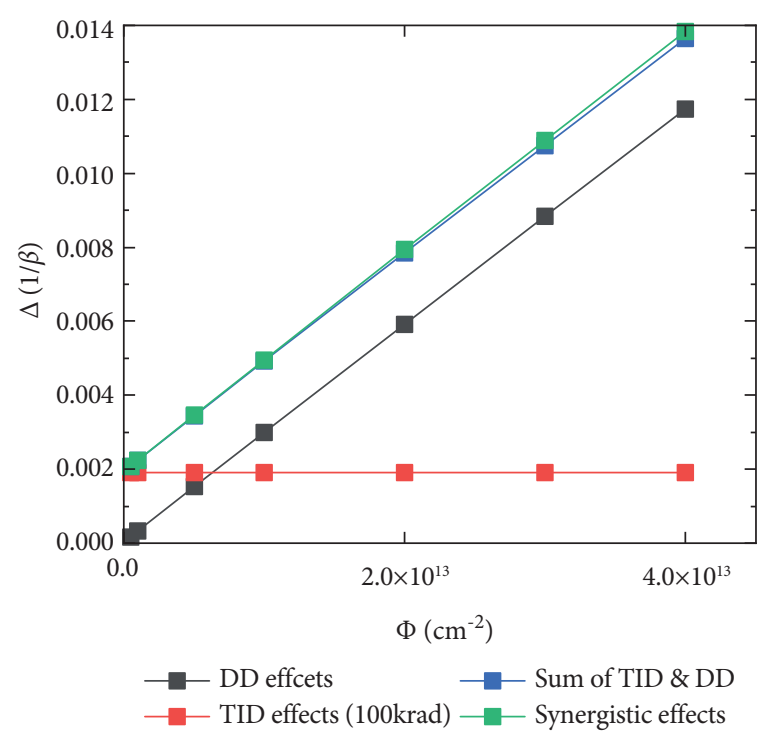

FIGURE 4: Synergistic effects of neutrons and $100 \mathrm{krad}$ gamma rays.

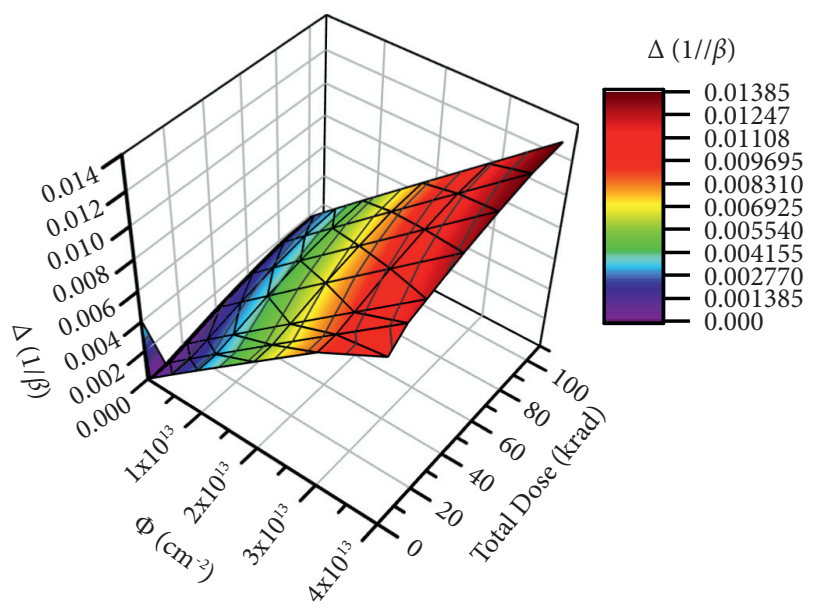

FIGURE 5: 3D figure of synergistic effects.

3.3. Analysis and Discussion. The sensitive area of the transistor to ionization damage and displacement damage is different. Figure 6 shows the recombination rate of carriers in the transistor: Figure 6(a) shows before radiation; Figure 6(b) shows irradiated with $100 \mathrm{krad}\left(\mathrm{SiO}_{2}\right)$ gamma rays; Figure 6(c) shows irradiated with $4 \times 10^{13} \mathrm{~cm}^{-2}$ neutrons; Figure 6(d) shows irradiated with both $100 \mathrm{krad}\left(\mathrm{SiO}_{2}\right)$ gamma rays and $4 \times 10^{13} \mathrm{~cm}^{-2}$ neutrons simultaneously. After comparison, the total recombination rate near the $\mathrm{Si} / \mathrm{SiO}_{2}$ interface is significantly higher than that before irradiation, which indicates that the interface traps enhance the surface recombination process and generates an excess base current. Neutron irradiation increases the total recombination rate in the base region of Si bulk. Although Figure 6(d) shows a promotion of carrier recombination in both interface and bulk, the discrepancy between Figure 6(d) and the combination of Figures 6(c) and 6(d) cannot be found apparently.

Figure 7 shows that the total carrier recombination rate from the interface to the Si bulk in the neutral base region, which 


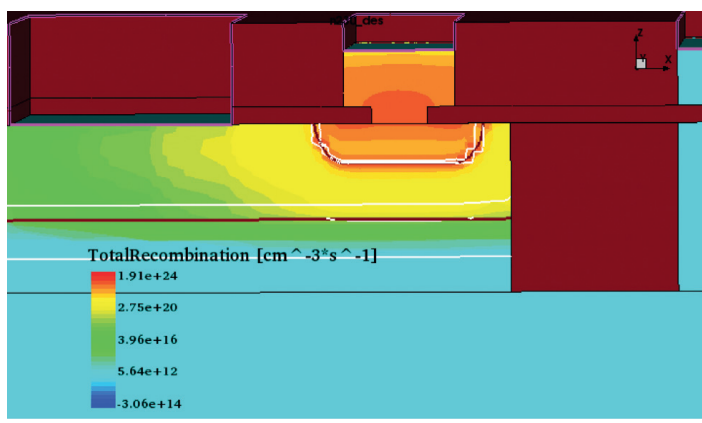

(a)

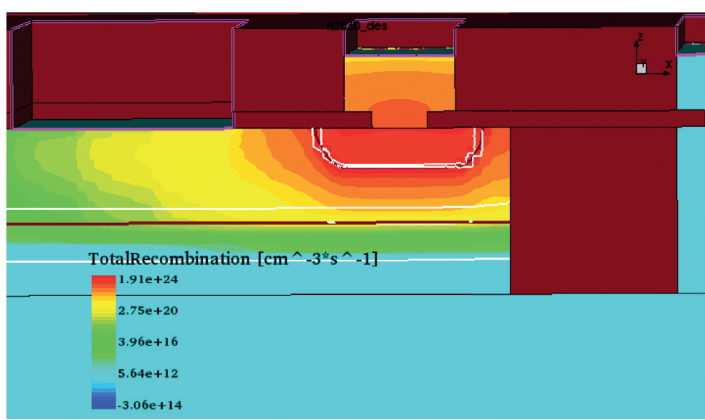

(c)

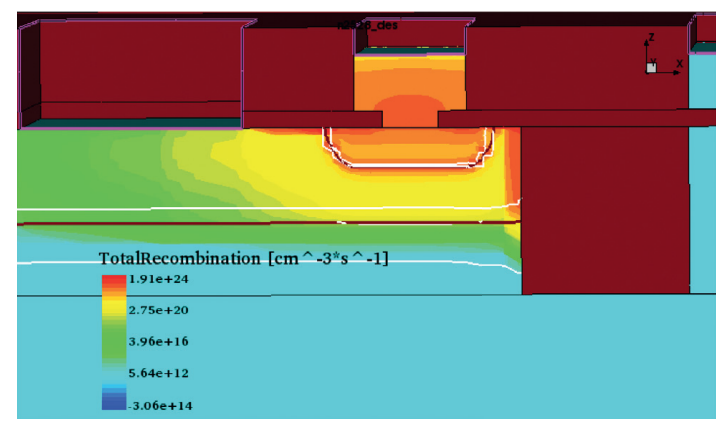

(b)

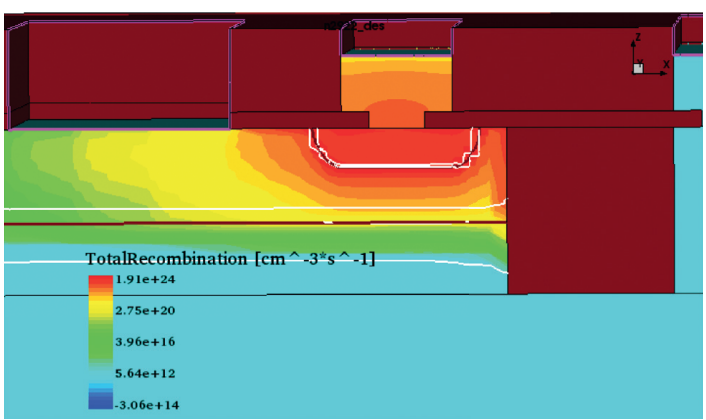

(d)

FIGURE 6: The recombination rate of carriers in the transistor. (a) Before radiation. (b) Irradiated with $\left.100 \mathrm{krad}^{(} \mathrm{SiO}_{2}\right)$ gamma rays. (c) Irradiated with $4 \times 10^{13} \mathrm{~cm}^{-2}$ neutrons. (d) Irradiated with both $100 \mathrm{krad}\left(\mathrm{SiO}_{2}\right)$ gamma rays and $4 \times 10^{13} \mathrm{~cm}^{-2}$ neutrons simultaneously.



FIGURE 7: Total carrier recombination rate from the interface to the bulk of silicon.

illustrates the mechanism of synergistic effects. As shown in the figure, the increase in surface recombination rate mainly occurs within the distance of $0.013 \mu \mathrm{m}$ to the interface, where the carriers are captured by the interface traps. Compared with TID and DD effects, the peak value of surface recombination rate is greater, and the recombination rate of Si bulk increases in the synergistic effects, which results in a higher recombination rate of carriers and more serious gain degradation.



FIGURE 8: The influence of DD effects on the carrier recombination rate of TID effects.

To explore the interaction between neutron irradiation and gamma rays irradiation, the neutron irradiation fluence and the total dose of gamma rays were controlled, respectively. Figure 8 shows the total recombination rate versus distance to the interface with various neutron fluence and fixed total dose of gamma rays at $100 \mathrm{krad}\left(\mathrm{SiO}_{2}\right)$. As the neutron fluence increases continually, the peak value of carrier surface recombination rate continues to rise, showing the promotion of DD effects to TID effects. Figure 9 shows 




FIGURE 9: The influence of TID effects on the carrier recombination rate of DD effects.

the total recombination rate versus various total doses of gamma rays with neutron fluence fixed at $4 \times 10^{13} \mathrm{~cm}^{-2}$. Although not obvious, it still shows the enhancement of TID effects on DD effects because of the increase of carrier's recombination rate in bulk $\mathrm{Si}$.

Researchers have proposed that the positive charges in the oxide layer promote the recombination of carriers in the defects of bulk $\mathrm{Si}[10]$. However, the performance of interface traps cannot be ignored. For NPN transistors, the interface traps are positively charged, which generated by the TID effects will reduce the difference in carrier's concentration between the $\mathrm{Si} / \mathrm{SiO}_{2}$ surface and the subsurface. The increase of the carrier recombination rate in the bulk Si leads to a decrease in the lifetime of electrons drifting and diffusing from the emitter, which are more likely to be captured by traps, leading to an increase in the peak value of the carrier coincidence rate on the interface.

3.4. Temperature Sensitivity. Transistors working in space are in various extreme temperature environments, which may make a different impact on the synergistic effects. The response of the synergistic effects of NPN transistors at different temperatures is simulated with the same conditions as in Section 3.2. As shown in Figure 10, the tendency of $\Delta(1 /$ $\beta$ ) of the synergy effect has not changed, but the lower the working temperature, the stronger the gain degradation caused by the synergy effect. On the contrary, the higher the temperature, the gentler the attenuation will be. Low temperature results in low carrier concentration and movement rate. Therefore, the device conductivity is poor, and the current under the same voltage is small, which has a great impact on the transistor gain; the opposite is true at high temperatures.

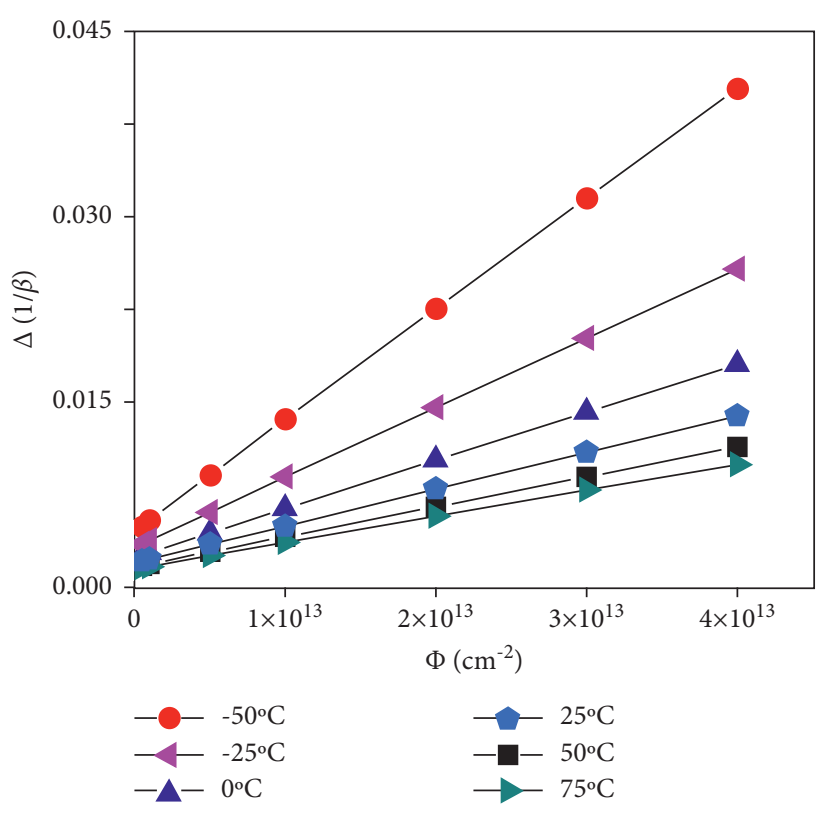

FIgURE 10: Synergistic effects of NPN transistors at different temperatures.

\section{Conclusions}

In summary, the synergistic effect of ionizing/displacement radiation to NPN bipolar transistors has been studied through building the three-dimensional model and simulation with TCAD. Since the gain degradation of the synergistic effects is greater than the sum of the TID effects and DD effects, the results of the simulation demonstrate the synergistic effects.

Analyzing the total carrier recombination rate of the device from the $\mathrm{Si} / \mathrm{SiO}_{2}$ interface to the bulk $\mathrm{Si}$, it is found that the mechanism of the synergistic effects is an interaction essentially between the DD effects induced by neutrons and the TID effects induced by gamma rays. Displacement damage enhances the peak of the total carrier recombination rate on the interface, and the interface traps enhance the carrier recombination rate in the bulk $\mathrm{Si}$ to a certain extent, resulting in synergistic effects overall. In addition, the influence of the device's working temperature on the synergistic effects is also explored. Compared with room temperature, a lower temperature will make the synergistic effects more serious.

According to the simulation results, the radiation experiments of ionizing/displacement synergistic effects are important for testing and evaluating the antiradiation performance of transistors. Keeping the device working at a constant temperature is vital for reducing radiation damage.

\section{Data Availability}

The data used to support the findings of this study are available from the corresponding author upon request.

\section{Conflicts of Interest}

The authors declare that there are no conflicts of interest regarding the publication of this paper. 


\section{Acknowledgments}

This work was supported by the National Natural Science Foundation of China (Grant nos. 61834004 and 61701505).

\section{References}

[1] X. Li, C. Liu, J. Yang, and G. Ma, "Research on the combined effects of ionization and displacement defects in NPN transistors based on deep level transient spectroscopy," IEEE Transactions on Nuclear Science, vol. 62, no. 2, pp. 555-564, 2015.

[2] X. Li, J. Yang, C. Liu, G. Bai, W. Luo, and P. Li, "Synergistic effects of NPN transistors caused by combined proton irradiations with different energies," Microelectronics Reliability, vol. 82, pp. 130-135, 2018.

[3] C. Wang, X. Bai, W. Chen et al., "Simulation of synergistic effects on lateral PNP bipolar transistors induced by neutron and gamma irradiation," Nuclear Instruments and Methods in Physics Research Section A: Accelerators, Spectrometers, Detectors and Associated Equipment, vol. 796, pp. 108-113, 2015.

[4] C. Wang, W. Chen, Y. Liu, X. Jin, S. Yang, and C. Qi, "The effects of gamma irradiation on neutron displacement sensitivity of lateral PNP bipolar transistors," Nuclear Instruments and Methods in Physics Research Section A: Accelerators, Spectrometers, Detectors and Associated Equipment, vol. 831, pp. 328-333, 2016.

[5] C. Wang, W. Chen, Z. Yao et al., "Ionizing/displacement synergistic effects induced by gamma and neutron irradiation in gate-controlled lateral PNP bipolar transistors," Nuclear Instruments and Methods in Physics Research Section A: Accelerators, Spectrometers, Detectors and Associated Equipment, vol. 831, pp. 322-327, 2016.

[6] G. Messenger and J. Spratt, "The effects of neutron irradiation on germanium and silicon," Proceedings of the Ire, vol. 46, no. 6, pp. 1038-1044, 1958.

[7] J. R. Srour, S. C. Chen, and S. Othmer, "Radiation damage coefficients for silicon depletion regions," IEEE Transactions on Nuclear Science, vol. 26, no. 6, pp. 4783-4791, 1980.

[8] O. M. Lawal, S. Liu, Z. Li, J. Yang, and A. Hussain, "Experimental studies of collector-emitter voltage bias influence on the total ionization dose effects in NPN Si BJTs," Superlattices and Microstructures, vol. 122, no. OCT, pp. 194-202, 2018.

[9] H. J. Barnaby, S. K. Smith, R. D. Schrimpf, D. M. Fleetwood, and R. L. Pease, "Analytical model for proton radiation effects in bipolar devices," IEEE Transactions on Nuclear Science, vol. 49, no. 6, pp. 2643-2649, 2002.

[10] H. J. Barnaby, R. D. Schrimpf, and A. L. Sternberg, "Proton radiation response mechanisms in bipolar analog circuits," IEEE Transactions on Nuclear Science, vol. 48, no. 6, pp. 2074-2080, 2002. 